\title{
Gastroenterology 2010: Maximising patient care and safety while minimising unnecessary cost
}

\author{
A symposium held on II November 2010 at the Royal College of Physicians of Edinburgh
}

CA Lamb

Wellcome Trust Clinical Research Fellow and Honorary Specialty Registrar in Gastroenterology, Newcastle University \& Newcastle upon Tyne Hospitals NHS Foundation Trust, UK

DECLARATION OF INTERESTS No conflict of interests declared.

\author{
Correspondence to CA Lamb, \\ Institute of Cellular Medicine, \\ The Medical School, Newcastle \\ University, Framlington Place, \\ Newcastle upon Tyne NE2 4HH, UK \\ tel. +44 (0)191 2820135 \\ e-mail c.a.lamb@ncl.ac.uk
}

In the light of challenging financial times affecting healthcare across the UK, the 2010 Gastroenterology Symposium focused on presenting the latest bestpractice evidence to effectively and safely manage patients with common gastrointestinal $(\mathrm{Gl})$ conditions while minimising unnecessary cost.

\section{SESSION I HOW DO I MANAGE?}

Professor Chris Probert (Professor of Gastroenterology, Bristol Royal Infirmary) outlined the clinical and financial problem of Clostridium difficile-associated diarrhoea (CDAD). Approximately 50,000 cases are diagnosed in England and Wales each year, with an annual cost to the European Union of $€ 3$ billion. Patients over 65 years are ten times more likely to develop CDAD due to increased hospitalisation, reduced immune response, higher prevalence of co-morbidity and greater prescription of antibiotics.' Central to reducing CDAD's health and cost burden is an early diagnosis and institution of effective treatment. Faecal enzyme-linked immunosorbent assay is currently the most common method of diagnosing CDAD but may miss as many as $20 \%$ of cases. In the future, gas chromatography mass spectrometry may provide a rapid, ward-based diagnosis method. Steps to improve CDAD prevention include the development of strict antibioticprescribing policies to avoid precipitating medications such as cephalosporins and fluoroquinolones, avoiding unnecessary hospital admission and discontinuing proton pump inhibitor therapy in high-risk patients.

Lower Gl bleeding is a common presenting problem experienced by up to one in seven adults. Miss Sarah Mills (Consultant Colorectal Surgeon,Wansbeck General Hospital, Northumberland) explained that although the vast majority will have no significant underlying pathology, colorectal cancer is the third most common malignancy in the UK and clinicians must consider each patient's risk. The concurrence of a change in bowel habit without anal symptoms (e.g. discomfort, prolapse or itching) should increase the clinical suspicion of cancer. ${ }^{2}$ Multiple endoscopic and radiological tests exist to investigate lower Gl bleeding, but the importance of a digital rectal examination at first clinic visit should not be underestimated. Haemorrhoids are a common cause of benign rectal bleeding. Most cases can be managed without specialist referral through dietary advice and strain avoidance.

Professor Chris Day (Pro Vice Chancellor, Faculty of Medical Sciences, Newcastle University) described the spectrum of non-alcoholic fatty liver disease from asymptomatic deranged liver function tests to liver cirrhosis, leading to hepatocellular cancer or the need for transplantation. Non-alcoholic fatty liver disease may be part of the metabolic syndrome, and is strongly associated with obesity, hypertension, diabetes and dyslipidaemia. Its prevalence may be more than $40 \%$ in the background population. ${ }^{3}$ The condition is an independent risk factor for cardiovascular disease, and is more likely to progress to end-stage liver disease in the presence of active hepatic inflammation/fibrosis. The key to treatment is lifestyle modification to lose weight and improve fitness. Potential medical therapies include vitamin E, pioglitazone for patients with type 2 diabetes mellitus and angiotensin-2 receptor blockers for those with hypertension.

\section{SESSION 2 ENSURING PATIENT SAFETY}

In the past ten years, biologics have revolutionised the management of complex inflammatory bowel disease (IBD). Despite their proven efficacy as powerful immunosuppressants, infliximab and adalimumab are not without complication risks. Professor Jack Satsangi (Professor of Gastroenterology, University of Edinburgh) discussed safety considerations such as potential side effects of sepsis, demyelination and malignancy, including hepatosplenic T-cell lymphoma and lung cancer. As IBD is a condition with associated morbidity but not mortality, the individual risk of a potentially life-threatening complication must be considered. Clinicians must be sure to exclude pelvic sepsis, screen for tuberculosis and consider alternative treatment strategies including surgery, where appropriate. Special care should be taken in older patients and in smokers.

Despite the emergence of biological therapy, steroids remain an important and commonly used therapeutic agent in gastroenterology. However, prolonged use can lead to osteoporosis.Professor Juliet Compston (Professor of Bone Medicine, University of Cambridge) explained that fracture risk increases with higher dosage steroids 
and longer duration of prescription. Bone protection must therefore be considered early in those who have received, or are going to receive, glucocorticoids for longer than three months. Knowledge of the ten-year probability of fracture is useful when planning treatment and can be calculated at www.shef.ac.uk/FRAX. Lifestyle measures to manage or avoid osteoporosis include maintaining adequate nutrition (calcium, vitamin $\mathrm{D}$ and normal body weight), avoiding smoking or excess alcohol and improved exercise. Pharmacological interventions in men and post-menopausal women include bisphosphonates or recombinant parathyroid hormone. In pre-menopausal women, calcium supplementation when dietary intake is low may be a more appropriate intervention.

\section{SESSION 3 GASTROINTESTINAL THERAPEUTICS, AVOIDING WASTE}

Dr Miles Parkes (Consultant Gastroenterologist, Addenbrooke's Hospital, Cambridge) reviewed the evidence base for using mesalazine in Crohn's disease. Whether given orally or rectally, mesalazine coats, and is metabolised by, intestinal epithelial cells, so the theoretical ability to penetrate transmural inflammation associated with Crohn's disease is questionable. Indeed, the current European Crohn's and Colitis Organisation consensus guidance on Crohn's disease states that 'mesalazine should be considered clinically no more effective than placebo for active ileal or colonic Crohn's disease' and '5ASAs are not recommended for maintenance of medically induced remission in Crohn's disease'. ${ }^{4}$ In these scenarios, clinicians should consider slow withdrawal for patients already receiving mesalazine. There may be a role for mesalazine in preventing recurrence in post-operative disease or as a chemopreventative agent in colorectal cancer associated with colonic Crohn's, but at present there is a lack of conclusive evidence to support this.

Dr Bjorn Rembacken (Consultant Endoscopist, Leeds Teaching Hospitals) outlined situations in which modern endoscopy may improve outcomes in pathologies that have historically been treated by surgery. Endoscopic mucosal resection (EMR) can be used in the stomach, colon, rectum or oesophagus to remove areas of mucosal dysplasia or even malignancy, provided it is well differentiated, superficial and has no lymphovascular invasion. Dr Rembacken also described the emerging treatment of endoscopic submucosal dissection which

\section{REFERENCES}

I Pépin J, Saheb N, Coulombe MA et al. Emergence of fluoroquinolones as the predominant risk factor for Clostridium difficile-associated diarrhea: a cohort study during an epidemic in Quebec. Clin Infect Dis 2005; 41:1254-60. doi:10.1086/496986

2 Thompson MR, Perera R, Senapati $A$ et al. Predictive value of common symptom combinations in diagnosing colorectal cancer Br J Surg 2007; 94:1260-5. doi:10.1002/bjs.5826

3 Williams $\mathrm{CH}$, Stengel J, Asike MI et al. Prevalence of nonalcoholic fatty liver disease and nonalcoholic steatohepatitis among a largely has the advantage of being able to remove large, nonpedunculated dysplastic or malignant polyps en bloc. Early results suggest that a complete removal of lesions may be possible in $85 \%$ of cases, but the perforation rate may be as high as $3.5-12 \%$ (vs $1.3 \%$ in EMR). ${ }^{5}$

Returning to the topic of patient safety, Mr Paul Philip (Deputy Chief Executive, General Medical Council [GMC]), explained the necessity for the GMC to introduce revalidation to ensure that all clinicians remain up to date and fit to practice. Revalidation will be a continual, formative process of assessing performance, ideally through existing appraisal frameworks and according to Good Medical Practice guidelines. A key outcome is to provide doctors with a non-threatening opportunity to reflect on the strengths and weaknesses in their practice.

\section{SESSION 4 NEW DEVELOPMENTS}

The past decade has seen major advances in the understanding of gene-environmental interactions that may lead to IBD. Dr Charlie Lees (Consultant Gastroenterologist, Western General Hospital, Edinburgh) explained that a total of 98 genes have now been identified as associated with either Crohn's disease, ulcerative colitis or both. These discoveries have greatly informed our understanding of disease pathogenesis. Perhaps in the future we may identify new immune targets or understand how best to use existing therapeutic agents by predicting response or complication risk according to a patient's genotype.

The symposium closed with an update on developments in the treatment of viral hepatitis from Dr Andrew Holt (Consultant Hepatologist, Queen Elizabeth Hospital, Birmingham) - please see his review on page 59.

\section{TAKE-HOME MESSAGE}

The UK is faced with a need to reduce public spending but has an obligation to facilitate the highest possible standards of patient care and safety. To achieve this, reducing disease burden through lifestyle modification and the restructuring of primary and secondary care services should be encouraged. Importantly, investment in translational research and the development of new technologies must not be neglected as they hold the potential to treat illness more quickly and safely in future.

middle-aged population utilizing ultrasound and liver biopsy: a prospective study. Gastroenterology 20I I; 140:124-31. doi:10.1053/j. gastro.2010.09.038

4 Dignass A, Van Assche G, Lindsay JO et al. The second European evidence-based consensus on the diagnosis and management of Crohn's disease: current management. J Crohn's Colitis 2010; 4:2862. doi:I0.1016/j.crohns.2009.12.002

5 Puli SR, Kakugawa Y, Saito Y et al. Successful complete cure en-bloc resection of large nonpedunculated colonic polyps by endoscopic submucosal dissection: a meta-analysis and systematic review. Ann Surg Oncol 2009; |6:2|47-5I. doi:I0.|245/s I0434-009-0520-7 